\title{
Retroperitoneal liposarcoma: an unusual case of weight gain despite weight loss measures
}

\begin{abstract}
Retroperitoneal liposarcomas are a heterogenous group of mesenchymal tumors that have a wide spectrum of histological subtypes and vague clinical presentation. ${ }^{1}$ They are locally invasive and the peak incidence is in the 5 th decade. ${ }^{2}$ They account for $0.1-0.2 \%$ of all solid tumors and $15 \%$ of all soft-tissue tumors. ${ }^{2}$ Liposarcomas are usually large and occur most frequently in the lower extremities, in the retroperitoneal, perineal and mesenteric region. ${ }^{2}$ In the retroperitoneum, they grow without symptoms due to the ability of the abdominal cavity to accommodate these slowly expanding masses. ${ }^{2}$ We present the case of $72 \mathrm{yr}$ old male who presented to urology and oncology due to the diagnosis of igh grade Gleason 9 prostate adenocarcinoma and a history of Agent Orange exposure. In the process of his workup, he complained of feeling full and gaining weight gain despite his best efforts at dieting and weight loss. Due to his abdominal distention, a CT scan of the abdomen and pelvis was performed which demonstrated 2 large fat containing intra abdominal masses suspicious for liposarcoma.
\end{abstract}

Keywords: retroperitoneal liposarcoma, abdominal masses, histological subtypes, weight gain

\author{
Volume II Issue 2 - 2020
}

\author{
Chintav Shah,' Simeon Jaggernauth, ${ }^{2}$ Vikram \\ A Ayapati, ${ }^{3}$ Blake o'Brian, 'Joseph Malhis' \\ 'Department of Internal Medicine, University of Arkansas for \\ Medical Sciences, USA \\ ${ }^{2}$ Landmark Cancer Center,Arkansas, USA \\ ${ }^{3}$ Medical student, USA
}

\section{Correspondence: Chintav Shah, Department of Internal Medicine, University of Arkansas for Medical Sciences,} Fayetteville,Arkansas, USA, Email chintavshah65@gmail.com

Received: April 02, 2020 | Published: April 15, 2020

\section{Background}

Liposarcoma is the most common primary retroperitoneal malignant neoplasm. ${ }^{3-5}$ The most frequent subtypes are liposarcoma (41\%), leiomyosarcoma (28\%), malignant fibrous histiocytoma (7\%), fibrosarcoma (6\%) and tumors of the peripheral nerve sheath (3\%). ${ }^{5}$ However, preoperative diagnosis is a common problem due to lack of characteristic clinical presentations. ${ }^{3}$ MRI is an ideal method for diagnosing retroperitoneal liposarcoma. ${ }^{3}$ Liposarcomas account for $30 \%$ of the retroperitoneal soft tissue sarcomas. ${ }^{4}$ Liposarcoma is the most common histopathological variety of the retroperitoneum.5 The average diameter of these tumors range from $20-25 \mathrm{~cm}$ with weight of $15-20 \mathrm{~kg} .^{5}$ There can be a compromise of the adjacent organs which is usually seen in $80 \%$ of the cases. ${ }^{5}$ Retroperitoneal liposarcoma is a distinct clinical entity that requires a more aggressive surgical approach which may include multiple resections or multi organ resection with recurrences. There is a low incidence of distant metastasis of approximately $7 \%$ compared to other histological subtypes that range from 15 to $34 \% .^{5}$

\section{Case report}

This is a 72year old male with an elevated BMI in the morbidly obease range and a history of Agent Orange exposure in Vietnam. $\mathrm{He}$ is a retired architect and presented initially to urology for a rising PSA. He has a history of a slowly rising PSA since 2010 which started out as 0.71 and rose to 4.1 in March 2019. He was seen by a urologist who performed a biopsy of the prostate which demonstrated evidence of a Gleason score $9(5+4)$ adenocarcinoma of the prostate involving $80 \%$ of the specimen of the left base.

During the process of his evaluation, the patient mentioned difficulty with failed attempts at weight loss through diet and exercise. A CT of the abdomen and pelvis was performed demonstrating evidence of 2 large fat containing intra-abdominal masses. One mass extended from the left upper quadrant into the left hemi pelvis and contained a thin nearly imperceptible wall with septation. A second similar but smaller mass was seen in the right of the midline extending from RUQ to the RLQ containing a $3 \mathrm{~cm}$ intensely enhancing mass along the anterior cephalad margin. The masses were thought to represent a lipoma vs liposarcoma. The prostate gland was noted to be enlarged. No retroperitoneal lymphadenopathy was seen and no osteolytic or osteoblastic lesions were identified. A CT guided biopsy of the abdominal mass was performed which showed findings consistent with liposarcoma. It was causing compression of the bowel lumen without associated pain, constipation or invasion of the visceral organs. He presented to medical oncology for further evaluation given the presence of these two malignancies (Figure $1 \& 2$ ).

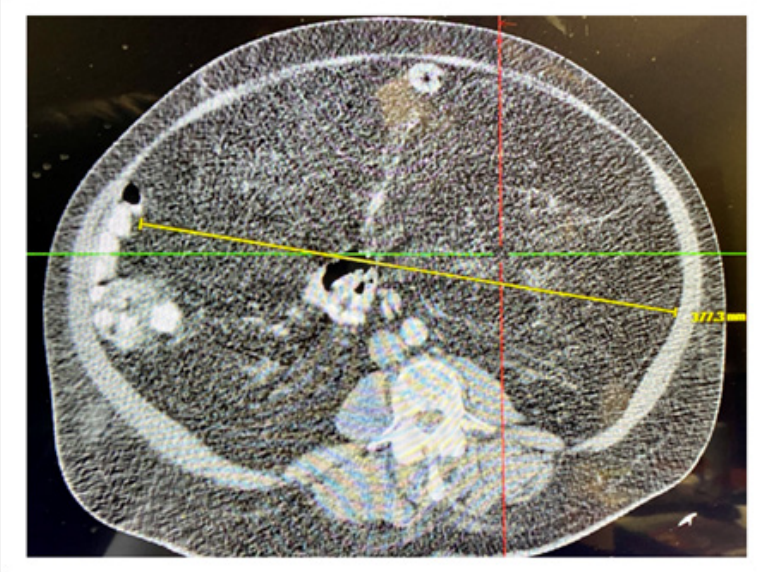

Figure I A CT scan of the abdomen. 


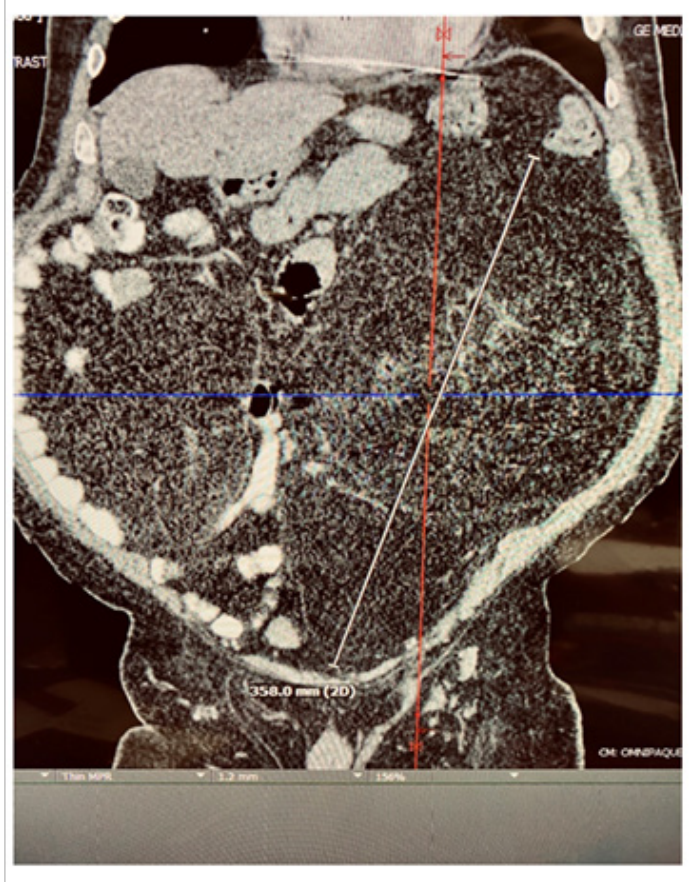

Figure 2 A CT scan of the pelvis.

Patient in the middle of all this workup also had a bone scan which demonstrated no scintigraphic evidence of osteoblastic metastases. The patient was then referred to UAMS and underwent surgical resection with pathology demonstrating evidence of a $46 \times 30 \times 18 \mathrm{~cm}$ well differentiated liposarcoma weighing approximately $14.3 \mathrm{~kg}$. His left kidney was also removed and no tumor invasion was identified. The second specimen measured $7.8 \times 6.0 \times 4.5 \mathrm{~cm}$. No adjuvant chemotherapy was required given the well differentiated low grade histology as well as the clear margins and no residual disease. He was able to start curative intent radiation therapy to the prostate now that the liposarcoma was out of the field of treatment with radiation.

\section{Discussion}

The single most important prognostic factor in patients with soft tissue sarcoma is the histologic grade of the primary lesion. ${ }^{2}$ As per
AJCC staging system, the grades are assigned from grade 1 (well differentiated) to grade 3 (poorly differentiated). In the treatment of the retroperitoneal sarcoma or genitourinary tract sarcoma, conventional chemotherapy does not seem to be effective, while radiotherapy has little improvement on the survival. ${ }^{2}$ Various chemotherapy regimens have been described based on mesna, doxorubicin, ifosfamide, dacarbazine, paclitaxel, pazopanib, and trabectedin. However, their use is limited for recurrent metastatic disease or palliation. ${ }^{5}$ The best treatment that still remains is surgical removal of the tumor. If there is no distant metastasis, patients will generally do well. The prognosis can be poor for liposarcomas with a $5 \mathrm{yr}$ overall survival of $15-50 \%$ for more aggressive histological subtypes. ${ }^{2}$ Because of the removal of his liposarcomas, he was able to start and complete curative intent radiation therapy for his highly aggressive prostate cancer.

\section{Acknowledgments}

None.

\section{Conflicts of interest}

The authors declare there are no conflicts of interest.

\section{Funding}

None.

\section{References}

1. Herzberg J, Niehaus K, Holl-Ulrich K, et al. Giant retroperitoneal liposarcoma: a case report and literature review. J Taibah Univ Med Sci. 2019;14(5):466-471.

2. Lauretti S, Cappa M, Emiliozzi P, et al. Retroperitoneal Liposarcoma. Minerva chirurgica. U.S. National Library of Medicine, 1998.

3. Song T, Shen J, Liang BL, et al. Retroperitoneal liposarcoma: MR characteristics and pathological correlative analysis. Abdom Imaging. 2007;32(5):668-674.

4. Cassinelli G, Arena E, Bronzino P, et al. Retroperitoneal Tumors: a Case of Liposarcoma. G Chir. 2002;23(11-12):420-422.

5. Herrera-Gómez A, Ortega-Gutiérrez C, Betancourt AM, et al. Giant Retroperitoneal Liposarcoma. World J Surg Oncol. 2008;6:115. 